\title{
Circular RNA hsa_circ_0072309 inhibits proliferation and invasion of breast cancer cells via targeting miR-492
}

This article was published in the following Dove Medical Press journal: Cancer Management and Research

Lin Yan'

Min Zheng'

Hua Wang ${ }^{2}$

'Department of Breast Surgery, The Second Affiliated Hospital and Yuying Children's Hospital of Wenzhou Medical University, Wenzhou 325000 , China; ${ }^{2}$ Department of Neurosurgery, The First Affiliated Hospital of Wenzhou Medical University, Wenzhou 325000, China
Correspondence: Hua Wang Department of Neurosurgery, The First Affiliated Hospital of Wenzhou Medical University, No.32, Dajian Lane, Lucheng District, Wenzhou 325000, China Email huawangh@I63.com
Background: Although the number of circular RNAs (circRNAs) that has been identified in multiple cancer tissues continues to increase, the relationship between circRNA expression and carcinogenesis remains unknown. The role of hsa_circ_0072309 in breast cancer has remained undefined until now. In this study, we aimed to investigate the role of hsa_circ_0072309 in breast cancer progression.

Methods: hsa_circ_0072309 expression in breast cancer tissues was analyzed using qRT-PCR. A series of functional experiments were carried out to investigate hsa_circ_0072309 function in breast cancer development and its underlying molecular mechanisms.

Results: hsa_circ_0072309 expression in breast cancer tissues was upregulated relative to that in adjacent normal tissues. hsa_circ_0072309 could serve as a prognostic biomarker of breast cancer. hsa_circ_0072309 overexpression dramatically inhibited the proliferation, migration, and invasion of breast cancer cells in vitro. In vivo assays revealed that the ectopic expression of hsa_circ_0072309 repressed breast cancer growth. The results of our mechanistic studies indicated that hsa_circ_0072309 could act as the sponge of miR-492, which exhibited increased expression in breast cancer tissues. Hsa_circ_0072309 suppressed breast cancer cell proliferation, migration, and invasion by inhibiting miR-492.

Conclusion: Our findings revealed for the first time that the hsa_circ_0072309-miR-492 axis plays an essential role in breast cancer progression.

Keywords: circular RNA, hsa_circ_0072309, miR-492, breast cancer, progression

\section{Introduction}

The incidence of breast cancer, the solid tumor class that causes the highest death rate among women worldwide, ${ }^{1,2}$ continues to increase. This malignancy will affect $12 \%$ of all women, and approximately $30 \%-40 \%$ of patients with breast cancer will die. ${ }^{3}$ Numerous therapies for breast cancer have been developed. These therapies, however, have demonstrated limited efficacy. Adjuvant therapy and hormonal treatment improve the survival rates and prognosis of patients with breast cancer but exhibit severe side effects that restrict their usage. ${ }^{3}$ Thus, methods for the early diagnosis, accurate prediction, and appropriate treatment of breast cancer still need to be developed. Understanding the molecular pathways involved in the pathogenesis of breast cancer is necessary to maximize treatment efficiency. ${ }^{4}$

Circular RNAs (circRNAs) are a group of non-coding RNAs that lack $5^{\prime}$ caps or $3^{\prime}$ poly-A tails. ${ }^{5}$ These non-coding RNAs were originally considered as splicing errors. ${ }^{6}$ Nevertheless, evidence showing that circRNAs are not simply the by-products of splicing errors and may regulate cellular function through distinct mechanisms continues to accu- 
mulate. ${ }^{7}$ Many circRNAs are dysregulated in cancer progression. ${ }^{8,9}$ Mechanistic studies have shown that circRNAs sponge miRNA expression and regulate biological processes. ${ }^{10,11}$ For example, circRNA ciRS-7 functions as an miRNA inhibitor by sponging miR-7. ${ }^{12}$ Information on the function of circRNAs in breast cancer progression, however, remains limited.

In this study, we investigated the function of hsa_ circ_0072309 in breast cancer progression. By using a public dataset (GSE77661), we found that circ_0072309 expression in breast cancer tissues was lower than that in normal control tissues. Thus, we selected this circRNA for further investigation. We discovered that hsa_circ_0072309 was downregulated in breast cancer cells and tumor tissues and that the downregulation of hsa_circ_0072309 was associated with the poor survival rate of patients with breast cancer. Overexpression experiments showed that hsa_circ_0072309 inhibited breast cancer progression in vitro and in vivo by regulating the proliferation of breast cancer cells. The results of our mechanistic studies revealed that hsa_circ_0072309 sponged miR-492. This effect consequently inhibited miR-492 and thus suppressed tumor progression. Our findings provide evidence that hsa_circ_0072309 plays an important role in breast cancer progression.

\section{Materials and methods}

\section{Patient specimens}

A total of 32 breast cancer tissues and matched normal tissues were obtained from The First Affiliated Hospital of Wenzhou Medical University. All tissue samples were stored in liquid nitrogen at $-80^{\circ} \mathrm{C}$ until use. Patients did not receive adjuvant therapy or endocrine therapy before surgery. Clinical features of the samples are listed in Table 1. This study was approved by the ethics committee of The First Affiliated Hospital of Wenzhou Medical University. Written informed consent was

Table I Clinicopathological features and hsa_circ_0072309 expression in 32 breast cancer patients

\begin{tabular}{|c|c|c|c|}
\hline Features & Low $(n=15)$ & High $(n=17)$ & $P$-value \\
\hline Age (years) & & & 0.287 \\
\hline$\leq 50$ & 6 & II & \\
\hline$>50$ & 9 & 6 & \\
\hline Tumor size $(\mathrm{cm})$ & & & $0.03 I^{*}$ \\
\hline$\leq 2$ & 5 & 13 & \\
\hline$>2$ & 10 & 4 & \\
\hline TNM staging & & & $0.032 *$ \\
\hline I-II & 4 & 12 & \\
\hline III-IV & 11 & 5 & \\
\hline Lymphoid node infiltrated & & & $0.036 *$ \\
\hline No & 3 & 10 & \\
\hline Yes & 12 & 7 & \\
\hline
\end{tabular}

Note: $* P<0.05$, statistically significant. obtained from all patients. Experiments involving human tissues were conducted in accordance with the Declaration of Helsinki.

\section{Cell culture and transfection}

Human breast cancer cell lines MCF-7 and T47D were obtained from the cell bank of the Chinese Academy of Science, Shanghai and maintained at $37^{\circ} \mathrm{C}$ in $5 \% \mathrm{CO}_{2} / 95 \%$ air in DMEM (Hyclone, Thermo Fisher Scientific, Waltham, MA, USA) containing 10\% FBS, 100 units/mL penicillin, and $100 \mu \mathrm{g} / \mathrm{mL}$ streptomycin.

MCF-7 and T47D cells stably overexpressing hsa_ circ_0072309 were constructed as previously reported. ${ }^{13}$ In brief, the coding sequence of hsa_circ_0072309 was inserted into the PLCDH-cir vector (Ribobio, Guangzhou, China). The lentiviral vector was constructed by Hanbio (Shanghai, China). Transfection was performed in accordance with the manufacturer's instructions. MCF-7 and T47D cells were selected with $0.5 \mu \mathrm{g} / \mathrm{mL}$ puromycin (Sigma-Aldrich Co., St Louis, MO, USA) for 2 weeks. MiR-492 mimics (5'-AGGACCUGCGGGACAAGAUUCUU-3') or negative controls were synthesized by Genepharma (Shanghai, China) and transfected into cell lines using Lipofectamine 2000 (Invitrogen, Thermo Fisher Scientific) following the manufacturer's instructions. Transfection efficiency was confirmed through qRT-PCR.

\section{Cell viability assay}

Cell viability was quantified with Cell Counting Kit-8 (CCK-8) (Beyotime, Jiangsu, China). MCF-7 and T47D cells were seeded in 96-well plates at the density of $2 \times 10^{3}$ cells per well. At different time points, $100 \mu \mathrm{L}$ of serum-free culture medium and $10 \mu \mathrm{L}$ of CCK- 8 solutions were added to each well. The plates were then incubated at $37^{\circ} \mathrm{C}$ for 1 hour. Absorbance was measured on ELX-800 spectrometer reader (Bio-Tek Instruments, Winooski, VT, USA) at $450 \mathrm{~nm}$.

\section{Colony formation assay}

A total of $1 \times 10^{3}$ cells were seeded in 6 -well plates. The medium was changed every 3 days. The cells were cultured until most colonies comprised more than 50 cells. Then, the cells were fixed with paraformaldehyde for 30 minutes and stained with $0.1 \%$ crystal violet. Finally, the number of colonies was counted.

\section{Flow cytometry analysis}

Cells were plated in 12 -well plates at the density of $1 \times 10^{6}$ cells per well. At 24 hours after incubation, the cells were fixed with ice-cold $70 \%$ ethanol and treated for 30 minutes with 1 unit of DNase-free RNase and $1 \mathrm{mg} / \mathrm{mL}$ propidium 
iodide at $37^{\circ} \mathrm{C}$. Then, $2 \times 10^{4}$ cells were analyzed with Arial III FACS system (BD Biosciences, San Jose, CA, USA).

\section{Luciferase assays}

The possible target miRNAs of hsa_circ_0072309 were predicted using the Circinteractome tool (https://circinteractome. nia.nih.gov). Sequences containing potential wild-type or mutant binding sites for miRNAs in hsa_circ_0072309 were constructed into pmirGLO vectors (Promega Corporation, Fitchburg, WI, USA). For the luciferase reporter assay, the reporter vector and miRNAs were co-transfected into MCF-7 cells using Lipofectamine 2000 (Invitrogen, Thermo Fisher Scientific). At 48 hours after induction, luciferase activity was measured using the dual-luciferase reporter assay system (Promega Corporation). Relative firefly luciferase activity was normalized to Renilla luciferase activity.

\section{Migration and invasion assays}

Migration and invasion assays were performed with chambers with pore sizes of $8 \mu \mathrm{m}$. For invasion assays, $3 \times 10^{4}$ cells were seeded into Matrigel-precoated chambers, and complete medium was added to the bottom of the wells to stimulate invasion. After 24 hours, the upper chambers were fixed with methanol and stained with crystal violet solution, and five fields (up, down, center, left, and right $\times 200$ ) were selected for the quantification of invading cells. The experimental procedure of the migration assay was the same as that of the invasion assay. The migration assay, however, was not performed with precoated chambers.

\section{qRT-PCR}

RNA was isolated with TRIzol reagent (Thermo Fisher Scientific), precipitated with phenol-chloroform and ethanol, and then resuspended in RNase-free $\mathrm{H}_{2} \mathrm{O}$. cDNA was prepared with PrimeScript RT reagent kit (TaKaRa, Japan) and subjected to qRT-PCR with ABI 7300 real-time PCR System (Applied Biosystems, Thermo Fisher Scientific). U6 and GAPDH were used as the internal controls.

\section{Tumor xenograft assay}

A total of $5 \times 10^{6}$ MCF-7 cells were subcutaneously injected into the right flanks of 4-week old female BALB/c (nu/nu) mice. A precision caliper was used to measure tumor size, which was recorded as the maximum diameter $(\mathrm{a}, \mathrm{mm})$ and vertical short diameter (b, $\mathrm{mm}$ ). Tumor volume was calculated using the formula $\mathrm{V}\left(\mathrm{mm}^{3}\right)=1 / 2 \mathrm{ab}^{2}$. At 4 weeks after injection, the mice were killed, and subcutaneous tumor weight was measured. Animal experiment protocols were approved by the Animal Ethics Committee of The First Affili- ated Hospital of Wenzhou Medical University. All animal operations were performed in accordance with the Animal Policy and Welfare Committee of The First Affiliated Hospital of Wenzhou Medical University.

\section{Statistical analyses}

SPSS 19.0 software (IBM Corporation, Armonk, NY, USA) was used for data analysis, and all data from three independent experiments were shown as mean \pm SD. Student's $t$-test was used to evaluate differences, and $P<0.05$ was considered as a statistically significant difference.

\section{Results}

\section{hsa_circ_0072309 was downregulated in breast cancer tissues}

CircRNAs, a large class of mammalian RNAs, have regulatory potential ${ }^{10}$ and essential roles in cancer development. ${ }^{9,14}$ Nevertheless, the functions of circRNAs have not been fully studied. We first analyzed hsa_circ_0072309 expression in breast cancer cell lines to identify the function of hsa_circ_0072309 in breast cancer carcinogenesis. Our qRT-PCR results showed that hsa circ_0072309 expression was downregulated in all six breast cancer cell lines relative to that in the normal breast cell line (Figure 1A). We further analyzed hsa_circ_0072309 expression levels in breast cancer tumor tissues. Our analytical results for the 32 pairs of tumor tissues and adjacent normal tissues show that hsa_circ_0072309 expression was downregulated in tumor tissues (Figure 1B). Specifically, hsa_circ_0072309 expression was decreased in more than $70 \%$ of patients with tumors (Figure 1C). In line with the downregulation of hsa circ_0072309 in breast cancer samples, survival analysis also showed that hsa_circ_0072309 downregulation was correlated with poor survival rates (Figure 1D). All these results indicate that hsa_circ_0072309 was downregulated in breast cancer.

\section{hsa_circ_0072309 overexpression inhibited breast cancer progression}

We then conducted functional assays to study the role of hsa_circ_0072309 in breast cancer carcinogenesis. We overexpressed hsa_circ_0072309 in MCF-7 and T47D breast cancer cell lines (Figure 2A) and analyzed the proliferation rates of hsa_circ_0072309-overexpressing cancer cells. Our CCK-8 assay results show that hsa_circ_0072309 overexpression inhibited MCF-7 and T47D cell proliferation (Figure 2B). This effect increased the number of cells in the G0/G1 stage (Figure 2C, D). Moreover, hsa_circ_0072309 overexpression reduced colony formation (Figure 2E).

Given that migration and invasion capabilities are characteristics of malignant cancer cells, we determined whether 
A

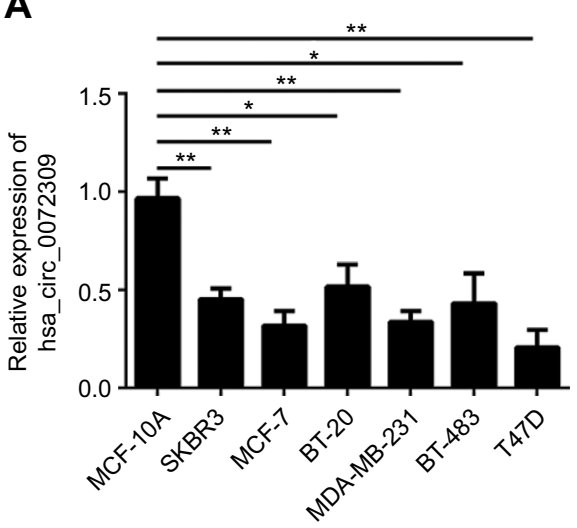

C

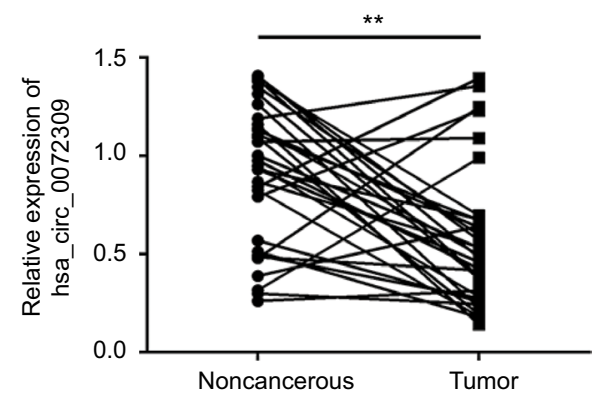

B

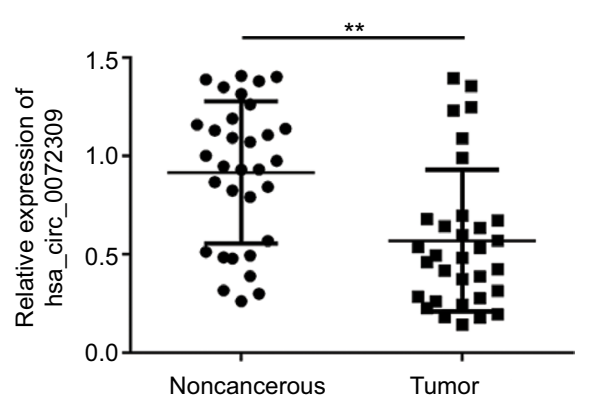

D

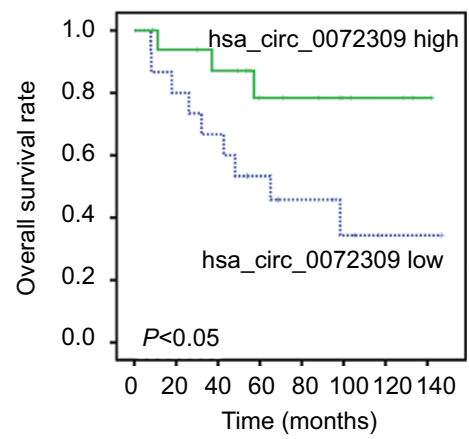

Figure I hsa_circ_0072309 was downregulated in breast cancer tissues.

Notes: (A) Relative expression of hsa_circ_0072309 in breast cancer cell lines was assessed by qRT-PCR. MCF-IOA is a noncancerous control cell line. Other cell lines are cancerous. (B) Relative expression of hsa circ 0072309 in 32 pairs of breast cancer tissues and adjacent noncancerous tissues. (C) Expression of hsa circ 0072309 was downregulated in the $78.13 \%$ (25/32) breast cancer tissues compared to adjacent normal tissues. (D) Overall survival curve for 32 breast cancer patients with high or low hsa_circ_0072309 expression by Kaplan-Meier analysis. $* P<0.05$ and $* * P<0.01$, vs control group. Every result was from three independent experiments.

hsa_circ_0072309 regulates the migration and invasion of breast cancer cells. The results of Transwell assays indicate that hsa_circ_0072309 overexpression inhibited the migration of MCF-7 and T47D cells (Figure 2F). In addition, the overexpression of hsa_circ_0072309 inhibited the invasion of MCF-7 and T47D cells (Figure 2G).

We knocked down hsa_circ_0072309 in MCF-7 and T47D cells to further investigate the physiological role of hsa_circ_0072309 (Figure S1A). The results of CCK-8 and Transwell assays show that hsa_circ_0072309 silencing enhanced the proliferation, migration, and invasion abilities of MCF-7 and T47D cells (Figure S1B-D). Thus, our data demonstrate that hsa_circ_0072309 overexpression suppressed breast cancer progression.

\section{hsa_circ_0072309 regulated breast cancer progression by acting as the sponge of miR-492}

CircRNAs function in cancer progression through several approaches, including sponging miRNA and regulating
RNA transcription and protein production. ${ }^{14}$ We first analyzed the subcellular location of hsa_circ_0072309 to study the molecular mechanism underlying its function in breast cancer. We found that hsa_circ_0072309 was mainly located in the cytoplasm (Figure 3A). Thus, we hypothesized that hsa_circ_0072309 may function as an miRNA sponge. Then, we conducted bioinformatics analysis to predict the binding partners of hsa_circ_0072309 and identify the ten most probable target miRNAs of hsa_circ_0072309 (Table 2). We performed luciferase reporter assays to validate our predicted results. Our results illustrate that luciferase activity was most intensely downregulated by miR-492 overexpression in MCF-7 cells (Figure 3B). Notably, the results of our CCK-8 assay indicate that miR-492 overexpression resulted in the most drastic increase in the proliferation potential of MCF-7 cells (Figure 3C). Thus, we selected miR-492 for further investigation. Our qRT-PCR results show that hsa_circ_0072309 overexpression downregulated miR492 expression (Figure 3D). Moreover, the deletion of the miR-492 binding site abrogated the suppressive effect of 

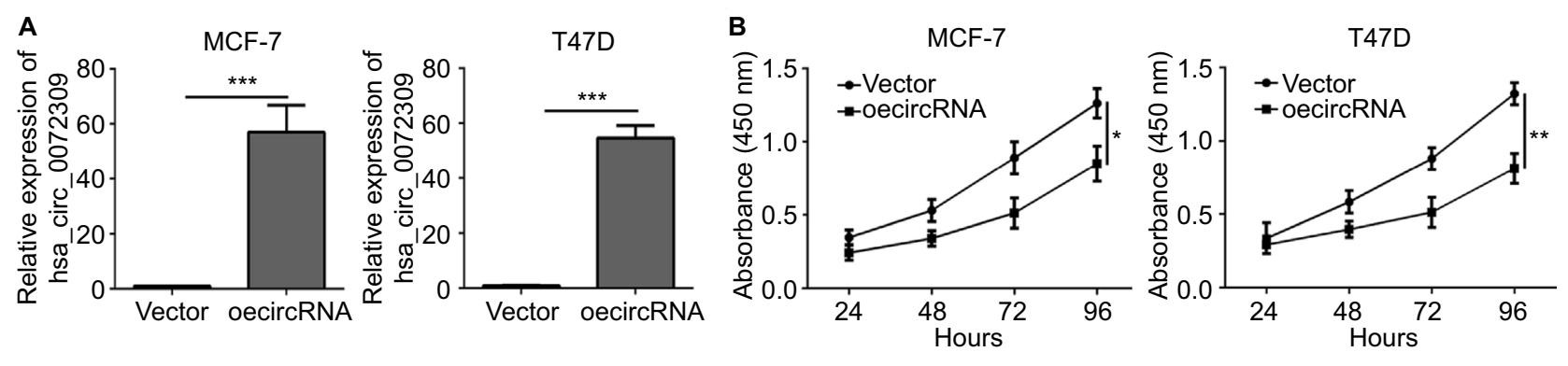

C
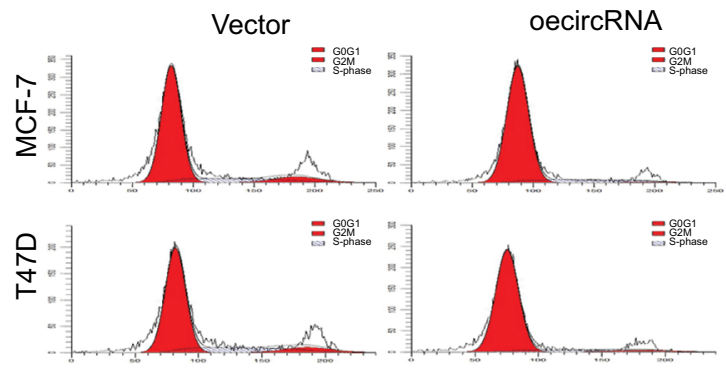

$$
\text { D }
$$
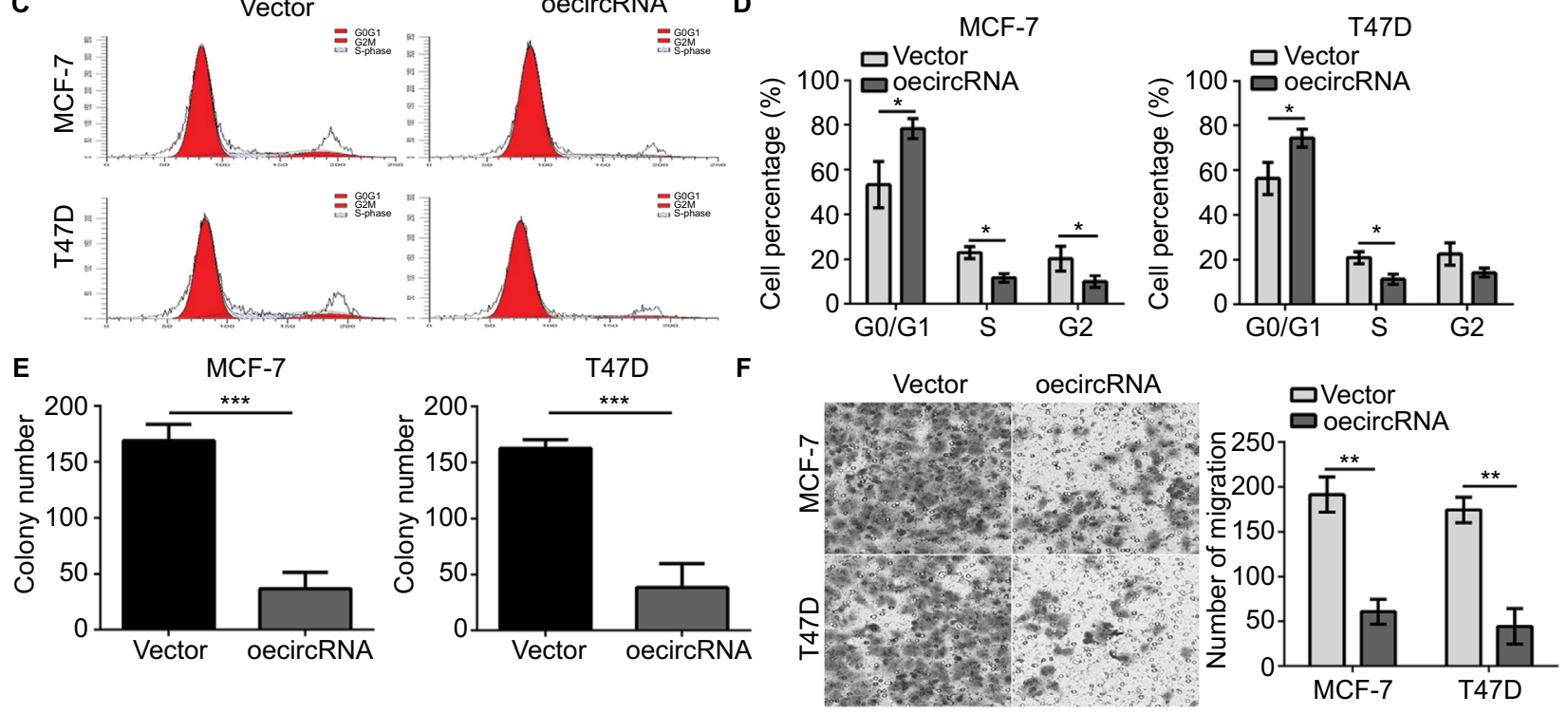

G

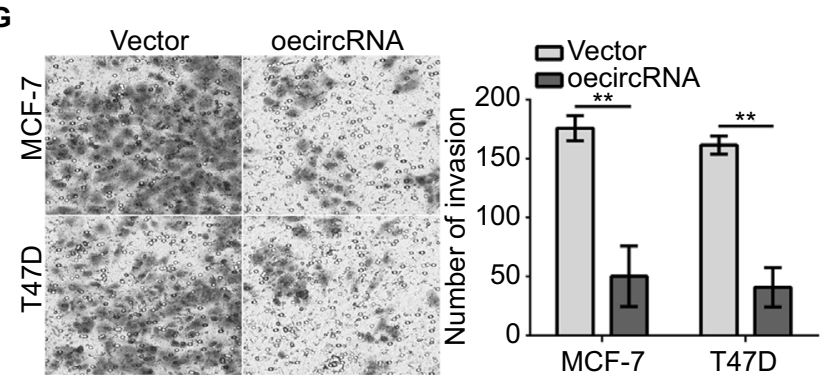

Figure 2 Overexpression of hsa_circ_0072309 inhibited breast cancer progression.

Notes: (A) Cells were transfected with vector or oe-hsa_circ_0072309. Forty-eight hours after transfection, qRT-PCR analysis was performed to determine hsa_ circ_0072309 expression. (B) Cell Counting Kit-8 proliferation assay indicated that the proliferation in hsa_circ_0072309 overexpression group was stronger than that in control group. (C, D) Cell cycle analysis was conducted by flow cytometry. (E) Colony formation assay showed that hsa_circ_0072309 overexpression inhibited breast cancer cell proliferation. (F) Transwell migration assay showed that hsa_circ_0072309 overexpression inhibited breast cancer cell migration. (G) Transwell invasion assay indicated that hsa_circ_0072309 overexpression reduced the invaded cell numbers. ${ }^{*} P<0.05$, $* * P<0.01$, and $* * * P<0.001$ vs control group. Every result was from three independent experiments.

hsa_circ_0072309 on miR-492 expression in MCF-7 and T47D cells (Figure 3E). This effect indicates that hsa_circ_0072309 and miR-492 interaction would inhibit miR-492 expression. Additionally, miR-492 expression was upregulated in breast cancer tissues (Figure 3F). All of these results prove that hsa_circ_0072309 is a sponge of miR-492.

Then, we investigated whether hsa_circ_0072309 promotes breast cancer progression through miR-492. We rescued miR-492 expression in hsa_circ_0072309-overexpressing MCF-7 and T47D cells (Figure 3G) and performed functional assays. Our results showed that the transfection of miR-492 mimics into hsa_circ_0072309-overexpressing cells rescued proliferation, migration, and invasion defects (Figure 3H-K) and thus restored normal cell cycle progression (Figure 3I). These results indicate that hsa_circ_0072309 inhibited breast cancer progression through miR-492. 

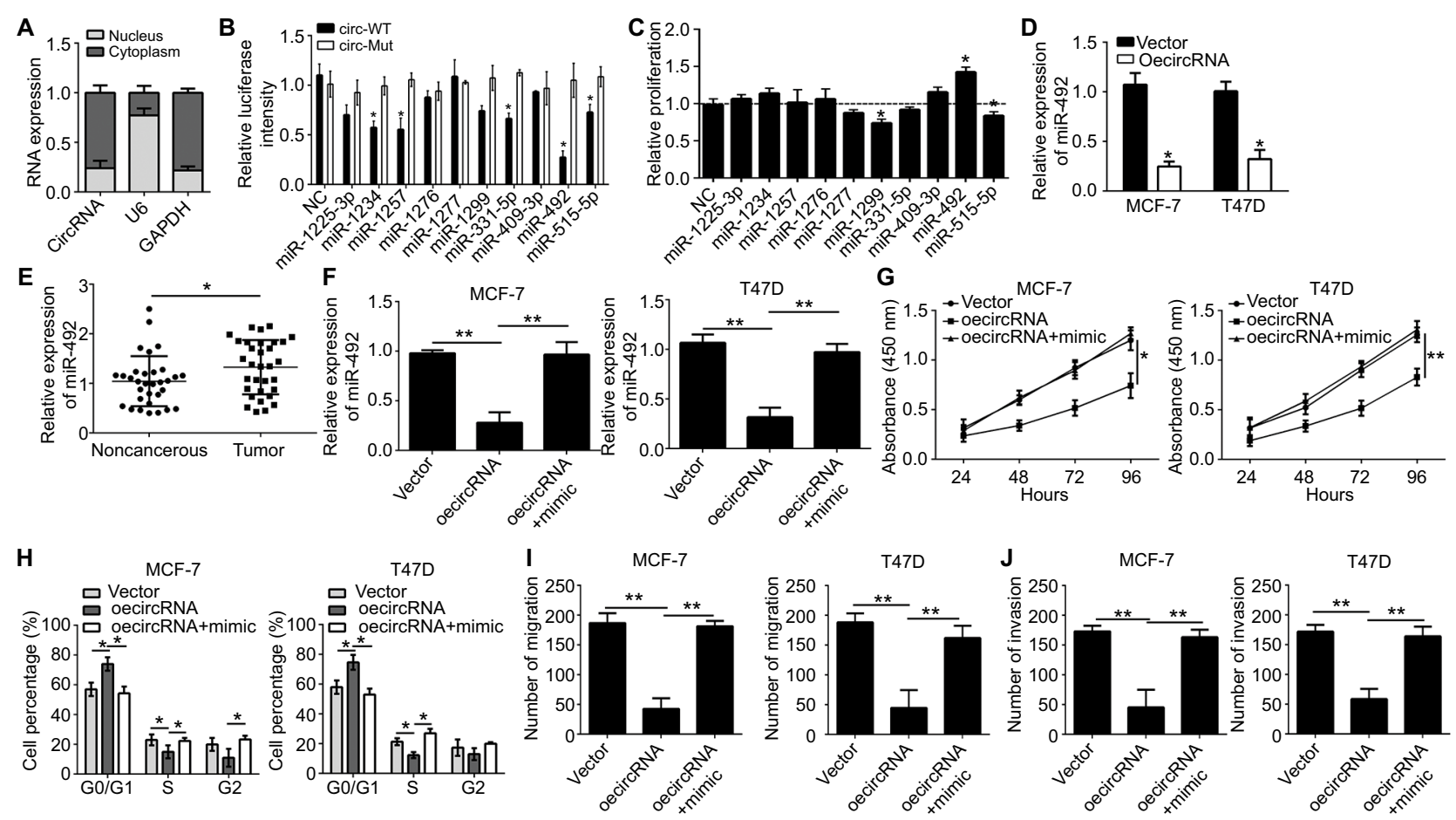

Figure 3 hsa_circ_0072309 was a sponge of miR-492 to regulate breast cancer progression.

Notes: (A) The levels of hsa_circ_0072309 were assessed by qRT-PCR in nuclear and cytoplasmic fractions. U6: nuclear control transcript; GAPDH: cytoplasmic control transcript. (B) Luciferase assays of MCF-7 cells co-transfected with indicated miRNAs and luciferase reporter containing hsa_circ_0072309-wild-type (WT) or mutant (Mut) construct. (C) CCK-8 assay was performed using MCF-7 cells. (D) Overexpression of hsa_circ_0072309 inhibited miR-492 expression in MCF-7 and T47D cells by qRT-PCR analysis. (E) MiR-492 expression was upregulated in breast cancer tissues compared to adjacent noncancerous tissues. (F) MiR-492 expression in MCF-7 and T47D cells transfected with oehsa_circ_0072309 and/or miR-492 mimic. (G) CCK-8 assay was performed to measure cell proliferation. (H) Cell cycle was determined by FACS analysis. $(\mathbf{I}, \mathbf{J})$ Transwell migration/invasion assays were utilized to test cell migration and invasion. $* P<0.05$ and $* * P<0.01$ vs control group. Every result was from three independent experiments.

Abbreviation: CCK-8, Cell Counting Kit-8.

Table 2 Target miRNAs of hsa_circ_0072309

\begin{tabular}{|l|l|}
\hline miRNA & Predicted binding sites \\
\hline miR-1225-3 $\mathrm{p}$ & $\begin{array}{l}\text { AAUAGCCAGAAAAAGGGGGCUCC } \\
\text { GACCCCCGCCGUGUCCCCGAGU }\end{array}$ \\
\hline miR-1234 & $\begin{array}{l}\text { AAGCACCCUCUGGAACAGGCCGU } \\
\text { CACCCCACCCACCAGUCCGGCU }\end{array}$ \\
\hline miR-1257 & $\begin{array}{l}\text { AAGUUCUACAAGUAAAUUCACA } \\
\text { CCAGUCUUGGGUAGUAAGUGA }\end{array}$ \\
\hline miR-1276 & $\begin{array}{l}\text { AGUAUUAAAAUUCCAGCUCUUUC } \\
\text { ACAGAGGUGUCCCGAGAAAU }\end{array}$ \\
\hline miR-1277 & $\begin{array}{l}\text { CUGGGAAAUUAAAGUUCUACGUA } \\
\text { UUUUAUGUAUAUAUAGAUGCAU }\end{array}$ \\
\hline miR-1299 & $\begin{array}{l}\text { AAAACGUUUCCUUAAUUCCAGAU } \\
\text { AGGGAGUGUGUCUUAAGGUCUU }\end{array}$ \\
\hline miR-331-5p & $\begin{array}{l}\text { CUCAACCUCUACAUUAUACCUAA } \\
\text { CCUAGGGACCCUGGUAUGGAUC }\end{array}$ \\
\hline miR-409-3p & $\begin{array}{l}\text { UCCAGUGGCUGUUAUCAACAUUU } \\
\text { UCCCCAAGUGGCUCGUUGUAAG }\end{array}$ \\
\hline miR-492 & $\begin{array}{l}\text { AGUUUGCAUUGAAAACAGGUCCC } \\
\text { UUCUUAGAACAGGGCGUCCAGGA }\end{array}$ \\
\hline miR-5I5-5p & $\begin{array}{l}\text { CGUUCUUGUUAUCAGUUGGAGAA } \\
\text { GUCUUUCACGAAAGAAAACCUCUU }\end{array}$ \\
\hline
\end{tabular}

Note: bold represents the binding sequence.

\section{hsa_circ_0072309 overexpression inhibited breast cancer growth in vivo}

From the previously mentioned results, we learned that hsa_circ_0072309 inhibited breast cancer progression in vitro. Thus, we investigated whether hsa_circ_0072309 inhibited breast cancer progression in vivo. We injected hsa_circ_0072309-overexpressing cells into nude mice and measured tumor growth every week. Our results indicate that hsa_circ_0072309 overexpression decreased the growth (Figure 4A) and sizes of breast cancer tumors (Figure 4B, C). These results show that hsa_circ_0072309 overexpression inhibited breast cancer progression in vivo.

\section{Discussion}

Information on the function of circRNAs in human disease remains limited. Similar to ncRNAs, circRNAs are expressed in multiple tissues and are highly diverse. Similar to tumor cells, different circRNAs exhibit distinctive 
A

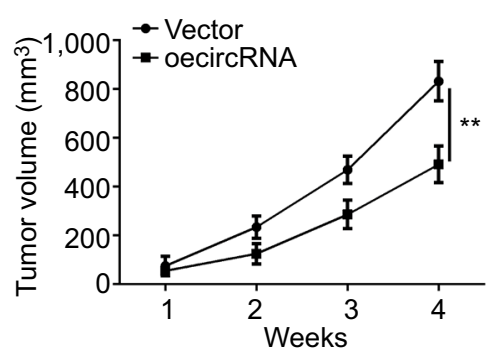

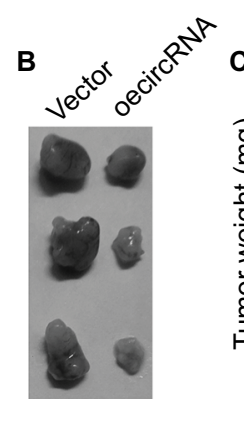

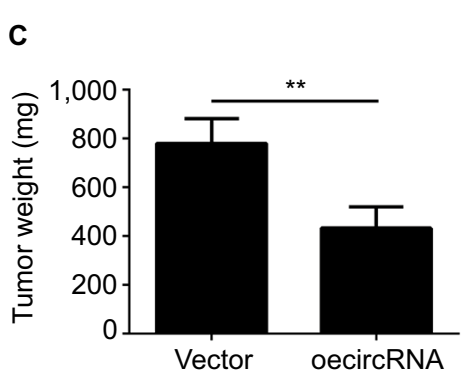

Figure 4 hsa_circ_0072309 overexpression inhibited breast cancer growth in vivo.

Notes: (A) Tumor volume was monitored every week for 4 weeks. hsa_circ_0072309-overexpressing or control MCF-7 cells were injected into the flank of the nude mice recipients. (B, C) Tumor photo and weights were measured and presented at the endpoint of xenograft experiments. ${ }^{* * P}<0.01$ vs control group. Every result was from three independent experiments.

expression patterns in various tissues and have specific functions in tumor progression. For example, circ_0001895 is downregulated in gastric cancer tissue and cell lines and is closely correlated with gastric cell differentiation. ${ }^{15}$ In contrast, circ_0000069 is upregulated in colorectal cancer and promotes colorectal cancer cell proliferation, migration, and invasion. ${ }^{16}$ These findings indicate that circRNAs have important regulatory roles in tumorigenesis. In this study, we found that hsa_circ_0072309 expression was downregulated in breast cancer tissues and cells. We preliminarily determined the biological function of hsa_circ_0072309 through further analysis.

The expression of different circRNAs is associated with the pathogenesis of breast cancer. ${ }^{17,18}$ Circ-ABCB10 is upregulated in breast cancer and promotes breast cancer progression. ${ }^{19}$ We discovered that hsa_circ_0072309 was downregulated in breast cancer tissues. Thus, the expression pattern of hsa_circ_0072309 opposes that of circ-ABCB10. In our study, we found that the downregulation of hsa circ_0072309 promoted the progression of breast cancer, and the expression of hsa_circ_0072309 was associated with the survival of breast cancer patients. Further functional assays showed that hsa_circ_0072309 overexpression inhibited MCF-7 and T47D cell proliferation, migration, and invasion. Thus, hsa_circ_0072309 functions as a tumor suppressor in breast cancer progression.

Similar to lncRNAs, circRNAs regulate miRNA expression in the cytoplasm by acting as miRNA sponges. ${ }^{20,21}$ The adsorption of circRNA to miRNA releases mRNAs from functional suppression by miRNA through reducing miRNA expression and would result in the formation of the circRNA-miRNA-mRNA regulatory axis. ${ }^{22}$ Cooperative communication between non-coding RNA and protein-coding RNA contributes to the biological complexities of cancer. ${ }^{23}$ Circ-MTO1 can suppress the progression of hepatocellular carcinoma through the circ-MTO1-miR-9-p21 axis. ${ }^{24}$ In the present study, we found that hsa_circ_0072309 inhibited breast cancer progression through miR-492 given that miR492 overexpression in hsa_circ_0072309-overexpressing cells rescued the failure of breast cancer progression. A previous study suggested that mIR-492 promotes the proliferation, migration, and invasion of breast cancer cells. ${ }^{25}$ Our study also demonstrated the oncogenic role of miR-492 in breast cancer.

Overall, we discovered that hsa_circ_0072309 is downregulated in breast cancer. Moreover, hsa_circ_0072309 functions as a tumor suppressor in breast cancer by inhibiting cell proliferation, migration, and invasion via miR-492. We discovered a critical hsa_circ_0072309-miR-492 function axis in breast cancer progression. Nevertheless, the function of miR-492 in breast cancer progression requires further investigation.

\section{Acknowledgment}

We thank all patients involved in this study.

\section{Disclosure}

The authors report no conflicts of interest in this work.

\section{References}

1. Elfar GA, Ebrahim MA, Elsherbiny NM, Eissa LA. Validity of Osteoprotegerin and Receptor Activator of NF- $\mathrm{kB}$ Ligand for the Detection of Bone Metastasis in Breast Cancer. Oncol Res. 2017;25(4):641-650.

2. Zhang Z, Wang J, Gao R, et al. Downregulation of MicroRNA-449 Promotes Migration and Invasion of Breast Cancer Cells by Targeting Tumor Protein D52 (TPD52. Oncol Res. 2017;25(5):753-761.

3. van Diest PJ, van der Wall E, Baak JP. Prognostic value of proliferation in invasive breast cancer: a review. J Clin Pathol. 2004;57(7):675-681.

4. Zhang N, Cao C, ZhuY, et al. Primary breast lymphoma: A single center study. Oncol Lett. 2017;13(2):1014-1018.

5. Meng S, Zhou H, Feng Z, et al. CircRNA: functions and properties of a novel potential biomarker for cancer. Mol Cancer. 2017;16(1):94.

6. Nigro JM, Cho KR, Fearon ER, et al. Scrambled exons. Cell. 1991;64(3):607-613. 
7. Chen JY, Hour TC, Yang SF, et al. Autophagy is deficient in nasal polyps: implications for the pathogenesis of the disease. Int Forum Allergy Rhinol. 2015;5(2):119-123.

8. Chen J, Li Y, Zheng Q, et al. Circular RNA profile identifies circPVT1 as a proliferative factor and prognostic marker in gastric cancer. Cancer Lett. 2017;388:208-219.

9. Kristensen LS, Hansen TB, Venø MT, Kjems J. Circular RNAs in cancer: opportunities and challenges in the field. Oncogene. 2018;37(5): $555-565$.

10. Memczak S, Jens M, Elefsinioti A, et al. Circular RNAs are a large class of animal RNAs with regulatory potency. Nature. 2013;495(7441): 333-338.

11. Hansen TB, Jensen TI, Clausen BH, et al. Natural RNA circles function as efficient microRNA sponges. Nature. 2013;495(7441):384-388.

12. Su C, Han Y, Zhang H, et al. CiRS-7 targeting miR-7 modulates the progression of non-small cell lung cancer in a manner dependent on NF-кB signalling. J Cell Mol Med. 2018;22(6):3097-3107.

13. Yang C, Yuan W, Yang X, et al. Circular RNA circ-ITCH inhibits bladder cancer progression by sponging miR-17/miR-224 and regulating $\mathrm{p} 21$, PTEN expression. Mol Cancer. 2018;17(1):19.

14. Li J, Yang J, Zhou P, et al. Circular RNAs in cancer: novel insights into origins, properties, functions and implications. Am J Cancer Res. 2015;5(2):472-480.

15. Shao Y, Chen L, Lu R, et al. Decreased expression of hsa_circ_0001895 in human gastric cancer and its clinical significances. Tumor Biol. 2017;39(4):101042831769912.

16. Guo JN, Li J, Zhu CL, et al. Comprehensive profile of differentially expressed circular RNAs reveals that hsa_circ_0000069 is upregulated and promotes cell proliferation, migration, and invasion in colorectal cancer. Onco Targets Ther. 2016;9:7451-7458.
17. Tang YY, Zhao P, Zou TN, et al. Circular RNA hsa_circ_0001982 Promotes Breast Cancer Cell Carcinogenesis Through Decreasing miR-143. DNA Cell Biol. 2017;36(11):901-908.

18. Liu Y, Lu C, Zhou Y, Zhang Z, Sun L. Circular RNA hsa_ circ_0008039 promotes breast cancer cell proliferation and migration by regulating miR-432-5p/E2F3 axis. Biochem Biophys Res Commun. 2018;502(3):358-363.

19. Liang HF, Zhang XZ, Liu BG, Jia GT, Li WL. Circular RNA circ$\mathrm{ABCB} 10$ promotes breast cancer proliferation and progression through sponging miR-1271. Am J Cancer Res. 2017;7(7):1566-1576.

20. Chen K, Lv X, Li W, et al. Autophagy Is a Protective Response to the Oxidative Damage to Endplate Chondrocytes in Intervertebral Disc: Implications for the Treatment of Degenerative Lumbar Disc. Oxid Med Cell Longev. 2017;2017(9859):1-9.

21. Dong Y, He D, Peng Z, et al. Circular RNAs in cancer: an emerging key player. J Hematol Oncol. 2017;10(1):2.

22. Rong D, Sun H, Li Z, et al. An emerging function of circRNA-miRNAsmRNA axis in human diseases. Oncotarget. 2017;8(42):73271-73281.

23. Farazi TA, Horlings HM, Ten Hoeve JJ, et al. MicroRNA sequence and expression analysis in breast tumors by deep sequencing. Cancer Res. 2011;71(13):4443-4453.

24. Han D, Li J, Wang H, et al. Circular RNA circMTO1 acts as the sponge of microRNA-9 to suppress hepatocellular carcinoma progression. Hepatology. 2017;66(4):1151-1164.

25. Shen F, Cai WS, Feng Z, et al. MiR-492 contributes to cell proliferation and cell cycle of human breast cancer cells by suppressing SOX7 expression. Tumour Biol. 2015;36(3):1913-1921. 


\section{Supplementary material}

A

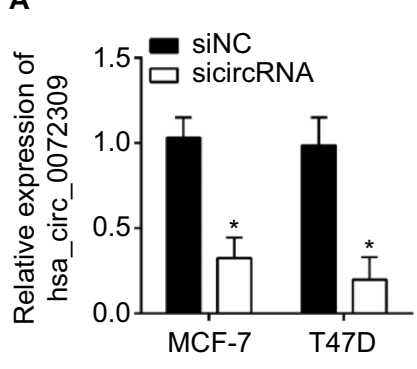

C

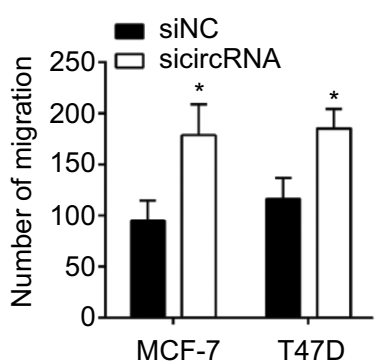

B

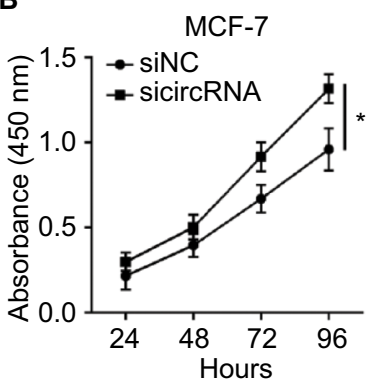

D

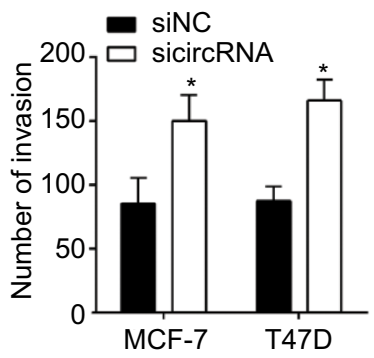

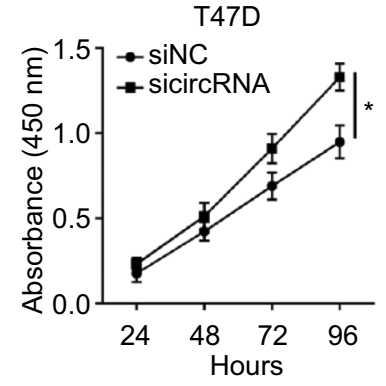

Figure SI hsa_circ_0072309 silencing promotes breast cancer cell proliferation, migration, and invasion.

Notes: (A) qRT-PCR showed hsa_circ_0072309 level was decreased after siRNA transfection. (B) CCK-8 assay showed that hsa_circ_0072309 silencing promoted proliferation. (C, D) Transwell assay showed that hsa_circ_0072309 silencing led to enhanced migration and invasion in MCF-7 and T47D cells. $* P<0.05$.

Abbreviation: CCK-8, Cell Counting Kit-8.

Cancer Management and Research

\section{Publish your work in this journal}

Cancer Management and Research is an international, peer-reviewed open access journal focusing on cancer research and the optimal use of preventative and integrated treatment interventions to achieve improved outcomes, enhanced survival and quality of life for the cancer patient. The manuscript management system is completely online and includes

a very quick and fair peer-review system, which is all easy to use. Visit http://www.dovepress.com/testimonials.php to read real quotes from published authors. 